In Human body, most important, complex and sensitive organ is Brain. Brain tumor is dangerous disease which threatens human life, hence detecting brain tumor in early stage plays an important part in treatment. Since Detection of Brain tumor is challenging task, medical imaging modality like Magnetic Resonance Imaging is helpful in diagnosis, analysis and treatment planning in brain tumor. MRI Images are processed by various pre-processing and post processing methods like Enhancement, Thresholding, Morphological operators, Segmentation etc through image processing tool in MATLAB. The Texture Features are extracted from the brain image by Feature Extraction methods such as Gray Level Co-occurrence Matrix, Fast Fourier Transform, Wavelet Transform etc and best feature is selected using any Feature Selection technique like Principal Component Analysis, Sequential Forward Selection, Particle Swarm Optimization etc. Thus important features are selected for comparison with stored database feature to classify between Normal and Abnormal images using Classifiers like Support Vector Machine, Artificial Neural Network, Probabilistic Neural Network, K-Nearest Neighbor, Machine Learning etc for detecting brain tumor

KEYWORDS: Human Brain, Brain Tumor, Magnetic Resonance Imaging, Image processing, Texture Feature, Feature Extraction, Feature Selection \& Image Classification

Received: Jun 10, 2017; Accepted: Jun 28, 2017; Published: Jul 05, 2017; Paper Id.: IJEEERAUG20172

\title{
INTRODUCTION
}

In human body, most complex organ is Brain. Human brain comprises billions of nerves, which communicates in trillions of connection known as Synapse. Human Brain controls each and every part of body and enables human to perform actions, gain knowledge, learn, share thoughts and communicate. Brain tissues are categorized into White Matter and Grey Matter. Grey Matter comprises of Glial cells and neurons which controls Brain activity. White Matter comprises of many axons[11]. Neurons are the important cells in human brain which has the capability to send signals to particular target cells by means of axons. Vertebrate brain has mainly six regions:

- Midbrain

- Cerebral hemispheres

- Diencephalon (thalamus and hypothalamus)

- Cerebellum 
- Medulla Oblongata

- Pons

[14]Often the midbrain, pons, and Medulla oblongata are referred to together as the brainstem. Brain stem is responsible for controlling functions like Breathing, sensory pathways, heart activity, reflexes etc. Cerebellum is responsible for controlling motor functions of human body like Walking, Posture, and Balancing etc. Brain contains Cerebrospinal fluid consisting of Enzymes, White blood cells, glucose and salt. To protect Brain and spinal cord from injury, Cerebrospinal fluid circulates through channels around them.

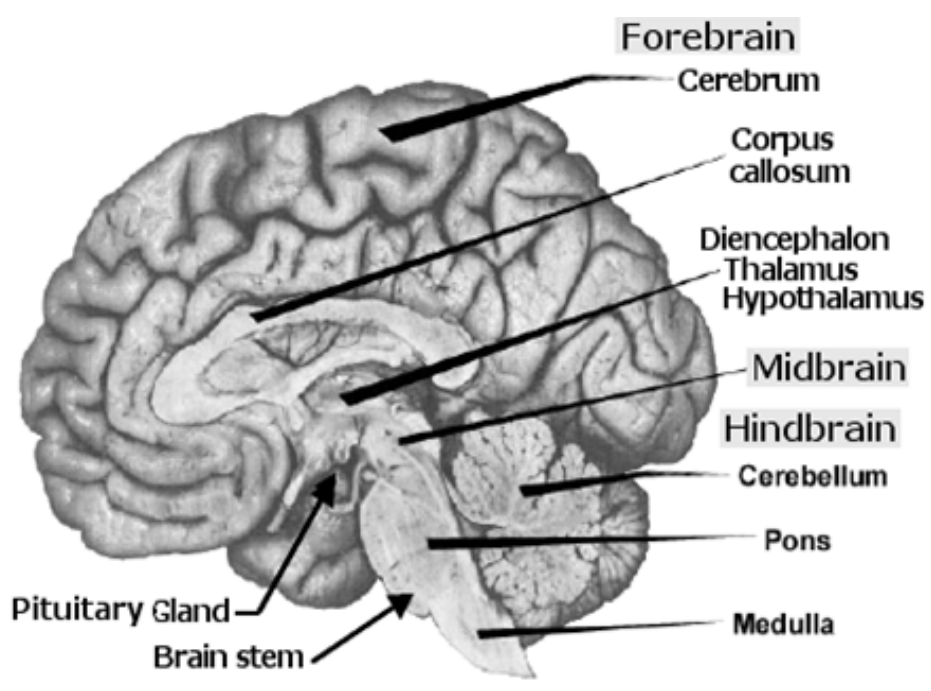

Figure 1: Human Brain and Regions

\section{Brain Tumor}

Brain tumor is composed of cells exhibiting uncontrolled and irregular growth in the brain. Brain Tumor is the abnormal mass of brain tissue occupying space in the skull and it is life threatening. Brain tumor interrupts normal functioning of brain and creates an increase in pressure causing shifting of some brain tissues. This results in damaging of nerves of other healthy brain tissues.

\section{There are Mainly Two Types of Tumor}

- $\quad$ Primary tumor

- Secondary tumor

Tumors that originate in the brain are called Primary Tumors. The Primary tumor can be Benign and Malignant. A Benign brain tumor (Non-cancerous) do not contain cancer cells and are slow growing. Benign tumor often has well-defined borders, so treatment can be done by surgically removing tumor. A Malignant tumor(cancerous) that grows and multiplies rapidly and may invade or destruct healthy areas of the brain. Malignant tumors are life-threatening, which spreads throughout the brain or to the spinal cord. Tumors originating from other parts of body and spread in brain are Secondary Tumors. Tumors can directly destroy all healthy brain cells. 


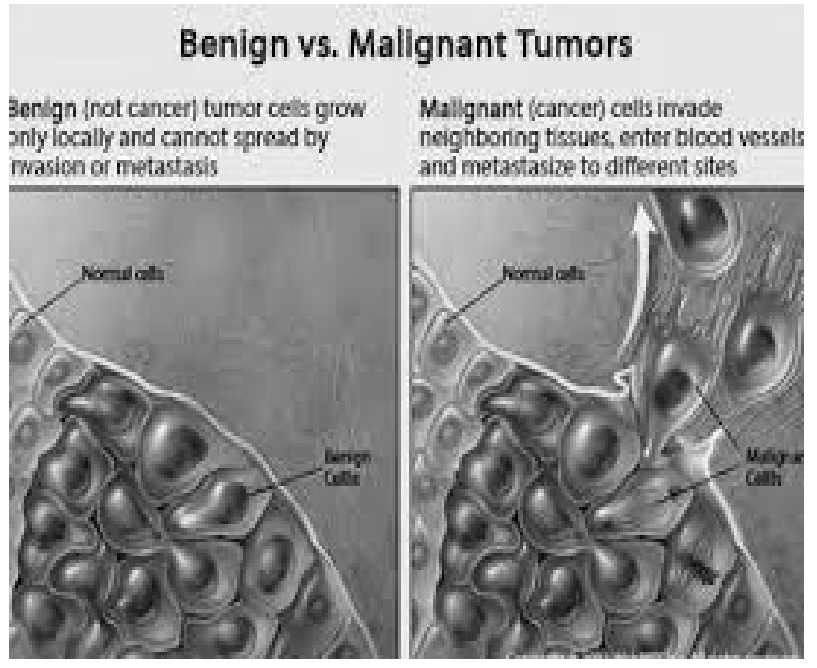

Figure 2: Types of Brain Tumor (Benign v/s Malignant)

In case where a brain tumor is suspected, patient undergoes a number of tests which helps the doctor reach to detect presence of tumor and determines the type of tumor. There are various diagnosing techniques which can be used for patients with neurologic conditions. Imaging techniques[12] such as:

Computed Tomography (CT) Scan: This scan uses an X-Ray-type device with a computer. The CT scanner circles the patient's head such that X-rays penetrate the brain from many directions.

Magnetic Resonance Imaging (MRI): MRI images are images which can be acquired on computer when a patient is scanned by MRI machine. Magnetic field generates signals and feed these signals into a computer creating a picture of the brain.

Positron Emission Tomography (PET): PET is not usually used for diagnosis, but it helps doctor in determining the grade of tumor.

Magnetic Resonance Spectroscopy (MRS): MRS is a diagnosing test that depicts the function rather than shape in images produced. MRS shows a pattern of activity which helps in diagnosing specific tumors and neurologic conditions.

\section{Magnetic Resonance Imaging}

Magnetic Resonance Imaging is a harmless and painless diagnosing test that uses radio waves and a magnetic field to produce images of the human organ with details. An MRI technologist performs Magnetic Resonance Imaging test on those patients that are suspected with a brain tumor. The MRI images are quite detailed, and are usually interpreted by a radiologist.

An MRI scanner is a tunnel-shaped piece of equipment that contains a strong magnet. For MRI test, the patient lies on a table that slides into the scanner. An MRI scanner uses a strong magnetic field, field gradients and radio waves which surround and produce images of the head. The MRI scan images are digital images which are saved and stored on a computer as records for further detailed study. 


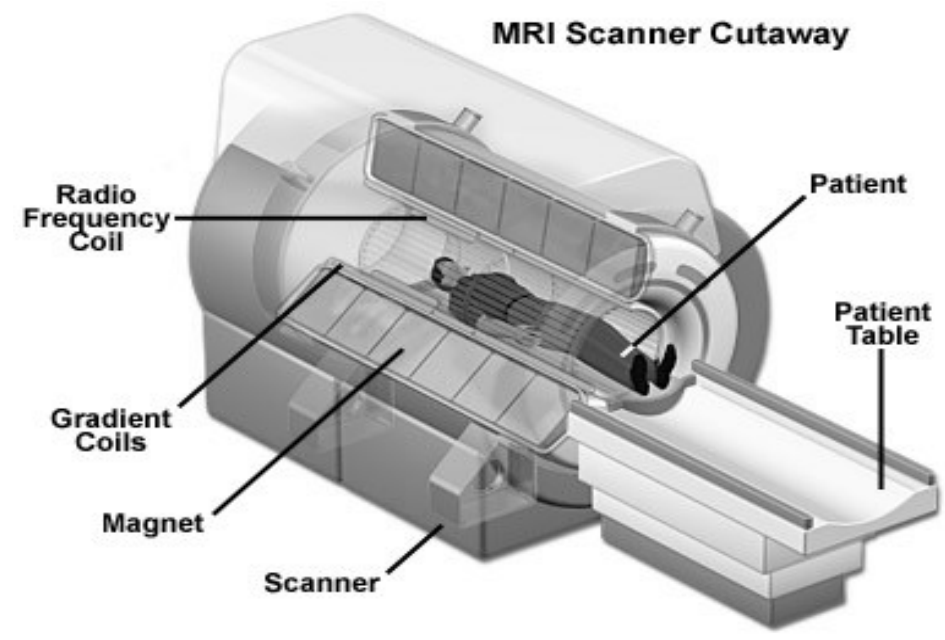

Figure 3: MRI Scanner

Magnetic Resonance Imaging is safe and non-invasive medical imaging modality test, which helps physicians to diagnose and treat medical conditions. Magnetic Resonance Imaging is based on Nuclear Magnetic Resonance. When an atomic nucleus is kept in a strong magnetic field, it absorbs and emits radio frequency energy. Examination of patient's head is performed in following three planes:

- Axial Plane MRI: It provides views of brain in a number of images starting from the chin towards the top of the head.

- Sagittal Plane MRI: It provides a view from the side of head in a series of images from one ear towards the other

- Coronal Plane MRI: It provides a view from behind the head in a series of images starting from the back of the head towards the face.

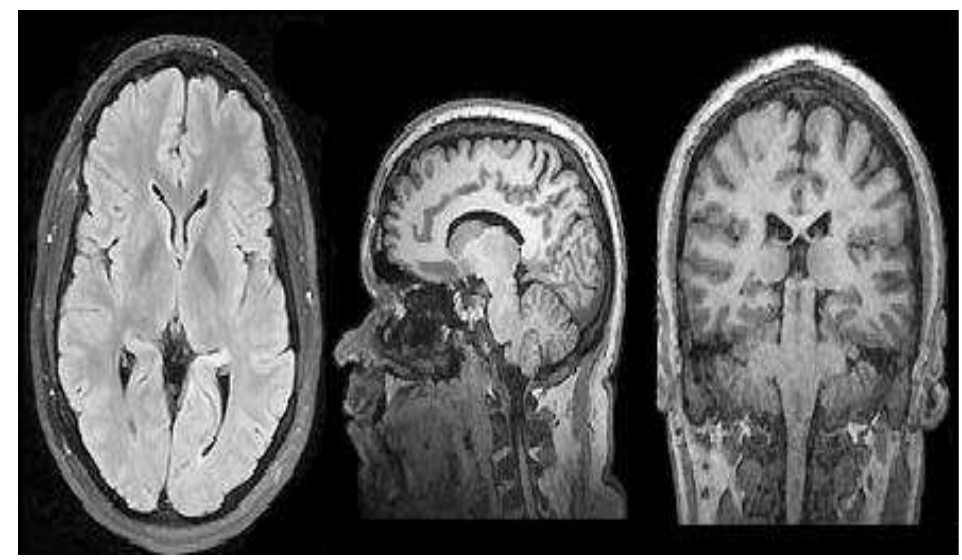

Figure 4: MRI Brain Image Plane (Axial, Sagittal, Coronal)

The Magnetic Resonance Image has Signal Intensity which is Determined by Four Basic Parameters

- Proton Density (PD): In the tissue, the concentration of protons are in the form of fats, proteins, water etc

- Longitudinal Relaxation Time (T1): After initial Radio Frequency pulse, the measure of how quickly the excited proton recovers to its equilibrium state

- Transverse Relaxation Time (T2): Rate at which excited protons fall to equilibrium (resting state) or go out of 
phase with each other.

- $\quad$ Flow: Loss of signal from rapid flowing arterial blood.

MRI brain images can be either Normal or Abnormal. The abnormal brain usually contains active tumor, blood clot, swelling, injury, infection etc. After digital images of brain are obtained, Image processing techniques can be further used for image enhancement followed by feature extraction, feature selection, image classification for the Brain tumor.

\section{RELATED WORK}

K. Mageswari, Dr. R. Renuga et. al. [5] proposed An Efficient Distributed Brain Image Classification via Particle Swarm Optimization and Support Vector Machine. The proposed system used the concept of distributed computing. The Features were extracted by the distribution system on the basis of Round robin scheduling followed by preprocessing. In this technique, feature selection is done by Particle Swarm Optimization and feature classification by Support Vector Machine (SVM). By using classifier, diseases like glioma, cerebral calcinosis, metastatic adenocarcinoma, carcinoma, sarcoma etc., from the abnormal brain images are identified efficiently.

Mohammed S. H. Tamimi and Ghazali Sulong et. al.[4] have presented Tumor Brain Detection Through MR Images: A Review of Literature. In this paper, some of the recent research works on detection of brain tumor and Segmentation using xMRI images were reviewed. The paper concludes with a concise discussion, providing a way towards the upcoming trend of more advanced studies and research on image segmentation and Tumor detection of brain.

N. Nandha Gopal, Dr. M. Karnan et. al. [2] have proposed Diagnose Brain Tumor Through MRI Using Image Processing Clustering Algorithms Such As Fuzzy C Means Along With Intelligent Optimization Techniques. In this paper, for diagnosing brain tumor through MRI using image clustering methods such as Fuzzy C Means with intelligent optimization tools, such as Genetic Algorithm and Particle Swarm Optimization were used to design an intelligent system. The tumor detection has two phases: Pre-processing and Image Enhancement in the first phase and segmentation and classification in the second phase.

Nikita V. Chauhan B.D. Jadhav, P.M. Patil et. al.[8] have proposed Detection and Classification of Brain Tumors. This paper elaborated an attempt for the brain tumor detection \& classification in benign stage. The proposed method includes two stages. In the first stage, texture features gets extracted by Gray Level Co-occurrence Matrix (GLCM) and in second stage, the image classification using K-nearest neighbor (K -NN) classifier.

P. Shantha Kumar and P. Ganesh Kumar et. al.[6] have developed Performance Analysis of Brain Tumor Diagnosis Based on Soft Computing Techniques, a new approach for automated detection of brain tumor. This proposed method includes pre-processing, anisotropic diffusion, extraction of features and classification. The gray level and wavelet features, local binary pattern are extracted. Then the features have been trained and classified by Support vector machine. The results obtained are quantitatively evaluated and compared with various ground truth images. This proposed method gives better and fast segmentation and classification rate gives sensitivity of $99.4 \%$, specificity of $99.6 \%$, positive predictive value of $97.03 \%$ and overall accuracy of $99.5 \%$.

Ahmed Kharrat, Karim Gasmi, Mohamed Ben Messaoud, Nacera Benamrane and Mohamed Abid et. al. [1] proposed A Hybrid Approach for Automatic Classification of Brain MRI into normal and benign or malignant classes using Genetic Algorithm and Support Vector Machine. This approach opts for genetic algorithm and support vector machine for 
classifying brain tissues through magnetic resonance images. For the extraction step we applied Spatial Gray Level Dependence Matrix to extract features. Features important for classification is selected using Genetic Algorithm. SVM classifier is used to classify the brain images into normal or abnormal(tumor). In proposed approach, experimental results show accuracy rate which varies from 94.44 to $98.14 \%$ while in the other approach accuracy rate varies from 92.59 to 96.29 $\%$.

S. Mahalakshmi and T. Velmurugan et. al. [10] did a research work on Detection of Brain Tumor by Particle Swarm Optimization using Image Segmentation. This research work analyses the detection of brain tumor through Magnetic Resonance brain images using Particle Swarm Optimization, a swarm intelligence based heuristic global optimization method. This work comprises of four phases which includes conversion, implementation, and extraction and selection.

Komal Sharma, Akwinder Kaur, Shruti Gujral et. al. [7] proposed Brain Tumor Detection based on Machine Learning Algorithms. Automated detection of tumor in Magnetic Resonance Imaging is very impotant because it gives information about abnormal(unheathy) tissues. In this paper, tumor is detected in brain MR images using machine learning algorithms. The proposed work consists of three phases: pre-processing steps are applied on brain MR images, texture features are extracted using Gray Level Co-occurrence Matrix and then machine learning algorithm is used for classification.

A.R. Kavitha et.al. [3] presented An Efficient Approach for Brain Tumor Detection Based on Modified Region Growing and Neural Network in MRI Images. The technique involves pre-processing, modified region growing, feature extraction and followed by classification. Evaluation of the performance of proposed technique is done considering the region growing algorithm and the modified region growing algorithm in terms of the quality rate. The tumor detection is evaluated through performance metrics namely, sensitivity, specificity and accuracy. Comparative analysis was made considering the normal and the modified region growing using both the Feed Forward Neural Network and Radial Basis Function neural network. From the metrics obtained, it is seen that the proposed technique proved its effectiveness by giving better results in terms of sensitivity, specificity and accuracy.

Ahmed Kharrat et. al. [9] presented MRI Brain Tumor Classification using Support Vector Machines and MetaHeuristic Method. They developed a new method for automated diagnosis, based on classification of Magnetic Resonance human brain images. For extracting feature, Spatial Gray Level Dependence Matrix with 2D Wavelet Transform were used. For reducing feature size, Simulated Annealing feature selection is applied. Then Stratified K-fold Cross Validation was used for avoiding over fitting. For optimization of support vector machine, Genetic Algorithm with Support Vector Machine (GA-SVM) model. SVM is applied to construct the classifier. An better classification rate of about $95 \%$ is achieved using the support vector machine.

\section{METHODOLOGY}

This paper presents a review on various techniques or steps used for the brain tumor detection using MRI brain images in Digital Image Processing [13], Detection of brain tumor consists of following stages:

- Image Acquisition

- Image Preprocessing

- Image Segmentation

- Feature Extraction 
- $\quad$ Feature Selection

- Image classification

\section{Image Acquisition}

For the purpose of Training, MRI image dataset can be collected from various repository sites and hospitals to form Database. The images can be in any format such as JPEG, DICOM etc. Size of each image is 512x512 having DICOM format. The images can be color or grayscale. A grayscale image is defined by using a large data matrix having entries of numerical values between 0 and 255 which represents shades of gray. The numeric value 0 represents to black and 255 white for instance.

\section{Image Preprocesssing:}

Prior to the analysis and extraction of desired features from original image, preprocessing of the acquired image dataset takes place. This includes correcting irregularities, removal of artifacts such as labels, highlighting the important portions of images, Noise removal, Reshaping, and RGB to Gray conversion, etc. After pre-processing, medical images are converted into images of improved quality without noise and artifacts. Image Enhancement is also an image processing method which involves Contrast enhancement, Edge Sharpening, Filtering etc. and suppresses noise.

\section{Image Segmentation:}

The Process by which digital image is partitioned into multiple number of segments i.e., set of pixels and separation of main part of brain image from unwanted pieces is called Image Segmentation. Images are divided and separated into many regions of similar features. Image Segmentation is used to locate boundaries and objects in image which simplifies the representation of image which is easier to analyze and more meaningful.

Thresholding: For every pixel of image, threshold value is calculated. Process of grouping all pixels of image into categories respective of their range of values in which the pixel lies. Thresholding is boundary approach image segmentation.

Region Growing: The process is based on intensity information in image. This is Region based approach image segmentation by selecting a seed point and extracting all pixels connected to it based on predefined condition.

Edge Based Segmentation: The Process of applying an edge filter which categorizes pixels of image as edge or non-edge depending on Filter output. This is an Edge based approach image segmentation.

\section{Feature Extraction}

Feature is a distinctive attribute i.e., instance with set of values that diverse the attribute from other. These are the properties which describe whole image. Features are the characteristics of image which defines the behavior of an image which shows its efficiency in classification. Since Analyzing input data with large number of variables need large computation power and memory which cause poor classification. Features are very important in Image processing. Features are mainly of following types:

- Shape Based Feature: Shape features are for real world object recognition and identification. The techniques for calculating shape features are Contour based and Region based feature extraction.

- Intensity Based Feature: Intensity features are extracted on absolute values. These features are related to intensity 
value of pixels in image

- Texture Based Feature: Texture features are for recognition and interpretation which can only be measured from a group of pixels. Texture features includes Correlation, Contrast, Entropy, Homogeneity, Energy, sum of square variance.

Feature Extraction is a technique applied to the input image to get features that will be useful in classifying and recognition of images. Feature Extraction is the process of reduction of data set by processing and computing certain properties that distinguishes any input pattern from other. There are different techniques to extract texture features from image by computing pixel statistics

Gray Level Co-Occurrence Matrix: GLCM is a two-dimensional matrix of number of gray levels represented in various number of rows and columns in the image that contains information about the position of pixel having similar gray level values. Gray level Co-occurrence matrices are calculated for four Orientations horizontal, right diagonal, vertical and left diagonal $\left(0^{\circ}, 45^{\circ}, 90^{\circ}\right.$ and $135^{\circ}$ degrees $)$

Gabor Filter: Gabor filter is used in Image Texture feature extraction. Sampling the complete frequency domain of image entirely by characterizing the center frequency and orientation parameters. Bank of Gabor filters (wavelet) are used to filter image. Each Gabor wavelet captures at particular frequency and direction that gives a localized frequency treated as a feature vector. From group of energy distribution, texture features can be extracted.

Discrete Wavelet Transform: The signal processing method by which data is processed. Discrete Wavelet Transform is mathematical tool for feature extraction from Magnetic Resonance images, which separates data into different frequency components. DWT is Linear transformation of data vector having a length of power of 2 into a numerical different vector having same length. It is popular for feature extraction producing a multi resolution image for interpreting information in image. Feature extraction using DWT involves Filtering and Decimation, Decomposition of selected image or signal into $\mathrm{N}$ level to obtain approximation and finer coefficient and extraction of features such as variance, mean, frequency etc.

Fast Fourier Transform: FFT is feature extraction technique which converts spatial domain into frequency domain. Image is represented in terms of intensities by its location and position in space $\mathrm{n}$ spatial domain. To use and represent image in its amplitude form, where amplitude values are stored in frequency domain.

\section{Feature Selection}

Images have excessive features not only increase computation time but also increase storage memory during classification. Since these features are redundant, little value can be added for classification. So selection among these features needs to be done. Feature selection is a search technique that searches for the best features among a number of features. Feature selection helps to select the most valuable feature, reduces redundancy from data, helps in improving performance and model interpretability. Optimal feature vectors of extracted feature are selected by various techniques such as

- Principal Component Analysis: It is one form of dimensionality reduction of wavelet coefficient. PCA finds linear lower dimensional representation of a given set of data such that it preserves the variance of reconstructed data.

- Linear Discriminant Analysis: It is the method used for machine learning and pattern recognition to find a linear arrangement of features that separates different classes. It is supervised linear discriminator which uses the class 
information and find a set of vectors that maximizes the between class scatter while minimizing within class scatter. Linear Discriminant Analysis limits the feature vectors leading to accuracy in classification, decrease in complexity and less computation time.

- Particle Swarm Optimization: It is a population based optimization algorithm. PSO is provided with some random particles and after that by updating generations, search for optimal continues. Each particle has its position adjusted depending upon distance from its own best position in search space. Performance of each particle is defined by Fitness Function. Fitness function is used to measure closeness of particle from global optimum depending on optimization problem.

- Sequential Forward selection: In this feature selection method, criterion value is computed. Feature with best value is selected from obtained features using the function. Then remaining features obtained from the image forms a pair of features. Best pair is selected from this pair of features and best feature. Following this, triplets of features were formed by these two best features and one of remaining features The procedure continues until a predefined number of feature gets selected.

\section{Image Classification:}

The process by which a given test sample is assigned a class on basis of information gained by classifier during training. Abnormality and diseases such as tumors can be detected using image classification. To classify MRI brain image into healthy brain or brain tumor, accuracy of this abnormality detection method must be very high since treatment planning depends on identification. The designed and developed classification system works in two phases namely Training Phase and Testing Phase.

- K-Nearest Neighbor: It is non-parametric pattern classification technique. K-nearest neighbor selects best K neighbors with shortest distance by computing the distance from unlabelled data (test sample) to every data point (training samples) using Euclidean distance. Euclidean distance is the measure of distance between test and training set samples. KNN is supervised classifier which yields good performance for optimal values of $\mathrm{K}$.

- Back Propagation Neural Network: It consist of one or more layers. Each layer in Neural network has one or more neurons. The neuron (perceptron) is a device with many inputs, one output, and an activation function. BPNN algorithm consists of following stages:

- Initialization of weights

- Feed forward

- Back propagating error

- Updation of weights and biases.

In the First stage, Assigning small random values.. After initialization of weights, each output unit will get an input signal and transmit it to each of hidden units. In each hidden unit, Activation function is calculated and send the signal to each output unit. For the given input signal, output unit forms the response of the net. If there I difference between response and actual output then an error is generated which is sent back to the input layer. Error adjusts the value of weights accordingly to achieve the desired response. 
- Support Vector Machine: It is supervised learning model associated with learning algorithms. This learning algorithm analyzes data for the purpose of classification and regression analysis. SVM builds a model to assign new samples to a certain class, such that samples of different classes are divided by clear gap.SVM is efficient enough to perform a non-linear classification using kernels. Support vector machine transforms or maps input space into a higher dimension feature space through a non-linear function and construct hyper-plane with maximum distance from the closest points of training set. SVM is discriminant classifier defined by a separating hyper-plane formally.

\section{CONCLUSIONS}

In this paper, several techniques and algorithm for detecting brain tumor in MR images have been discussed. This paper provides a review of all image processing, feature extraction, feature selection and classification methods that are performed on different MR images. Each and every method differs in their algorithm and performance. Several methods can be combined to develop many hybrid approaches for better results in detection of brain tumor.

\section{REFERENCES}

1. Ahmed Kharrat, Karim Gasmi, Mohamed Ben Messaoud, Nacera Benamrane and Mohamed Abid, "A Hybrid Approach for Automatic Classification of Brain MRI Using Genetic Algorithm and Support Vector Machine” Leonardo Journal of Sciences ISSN 1583-0233 Issue 17, July-December 2010

2. Gopal, N.N. Karnan, M., -Diagnose brain tumor through MRI using image processing clustering algorithms such as Fuzzy C Means along with intelligent optimization techniques Page(s): 1 - 4, Computational Intelligence and Computing Research (ICCIC), 2010 IEEE International Conference, 28-29 Dec. 2010.

3. A.R. Kavitha, Dr. C. Chellamuthu, Ms. Kavin Rupa, "An Efficient Approach for Brain Tumour Detection Based on Modified Region Growing and Neural Network in MRI Images" 2012 International Conference on Computing, Electronics and Electrical Technologies [ICCEET] 978-1-4673-0210-4112 @2012 IEEE

4. Mohammed Sabbih Hamoud Al-Tammi, Ghazali Sulong, "Tumor Brain Detection Through MR Images: A Review of Literature ”Journal of Theoretical and Applied Information Technology ISSN:1992 8645. Vol. 62 No.2 April 2014

5. K. Mageswari, Dr.R.Renuga.2014"An Efficient Distributed Brain Image Classification via Particle Swarm Optimization and Support Vector Machine", International Journal of Scientific \& Engineering Research, Volume 5, Issue 4, April-2014. ISSN $2229-5518$.

6. P. Shantha Kumar and P. Ganesh Kumar, "Performance Analysis of Brain Tumor Diagnosis Based on Soft Computing Techniques", American Journal of Applied Sciences 11 (2): 329-336, 2014, ISSN: 1546-9239

7. Komal Sharma, Akwinder Kaur, Shruti Gujral," Brain Tumor Detection based on Machine Learning Algorithms" ISBN 978-81-929648-0-5 IRISET@2014, 2nd International Conference on Computer and Intelligent Systems (ICCIS’2014) \& 2nd International Conference of Electrical, Electronics, Instrumentation and Biomedical Engineering (ICEEIB'2014) May 30-31, 2014 Bangkok (Thailand)

8. Nikita. V. Chauhan, B. D. Jadhav, P. M. Patil. 2015. "Detection and Classification of Brain Tumors", International Journal of Computer Applications (0975 - 8887) Volume 112 - No. 8, February 2015

9. Ahmed Kharrat, Mohamed Ben Halimal, Mounir Ben Ayed, "MRI Brain Tumor Classification using Support Vector Machines and Meta-Heuristic Method” 2015 15th International Conference on Intelligent Systems Design and Applications (ISDA) 978-1-4673-8709-5/15/\$31.00, 2015 IEEE

10. S. Mahalakshmi and T. Velmurugan. 2015. "Detection of Brain Tumor by Particle Swarm Optimization using Image 
Segmentation”, Indian Journal Of Science and Technology Vol 8(22), DOI: 10.17485/ijst/2015/v8i22/79092 September 2015

11. http://www.spineneuro.org/anatomy-brain.shtml

12. http://www.studypk.com/articles/types-brain-scans/

13. Gonzalez, R.C. and Woods, R.E., "Digital Image Processing”, Addison-Wesley (USA), pp. 413-431, 1993

14. http://donsnotes.com/science/biology/neuro_anatomy.html 
